\title{
Actitud del personal profesional de Enfermería frente a la voluntad anticipada de la persona sobre su cuidado al final de la vida: caso de Costa Rica, 2011 ${ }^{1}$
}

Institución: Universidad de Costa Rica

\author{
Jerik Andrade Espinales ${ }^{2}$ \\ Viviana García Rojas ${ }^{3}$ \\ Daniel Martínez Esquivel ${ }^{4}$ \\ Carlos Miranda Otárola ${ }^{5}$ \\ Marilyn Quesada Morera ${ }^{6}$ \\ Katherine Vargas Villalobos ${ }^{7}$
}

\section{COMO CITAR}

Andrade, J.; García, V.; Martinez, D.; Miranda, C.; Quesada, M.; Vargas, K. (2012) Actitud de la enfermera (o) frente a la voluntad anticipada de la persona sobre su cuidado al final de la vida: caso de Costa Rica, 2011. Revista Enfermería Actual en Costa Rica. 23, 1-14. Recuperado de: http://www.revenf.ucr.ac.cr/voluntad.pdf. ISSN 1409-4568

\section{Resumen}

El problema de esta investigación fue analizar la actitud asumida por el profesional en Enfermería frente a la voluntad anticipada sobre el cuidado de las personas al final de la vida en Costa Rica durante el año 2011. Se aplicó una metodología cuantitativa de tipo exploratorio y transeccional con un muestreo probabilístico por conglomerados y un submuestreo polietápico posterior que tomó en cuenta los hospitales Nacionales clase A. Se obtuvo una muestra de 86 participantes, a la cual se le aplicó un cuestionario. Los datos obtenidos se tabularon por medio del paquete estadístico Statistical Package for the Social Sciences (SPSS). Estos reflejaron que, aunque la mayoría de participantes desconocen el concepto de voluntad anticipada, la relacionan con el respeto a la dignidad, los derechos y la autonomía como principios que predominan en la ética al momento de brindar cuidado. Se concluye que los enfermeros y las enfermeras muestran una actitud favorable hacia estos principios, los cuales priman en los roles que ejercen por sobre su propia ética y moral.

Palabras clave: actitud, Bioética, cuidado, Enfermería, moral, final-de-la- vida, voluntad-anticipada.

\footnotetext{
${ }^{1}$ Fecha de recepción: 10 de marzo 2012

Fecha de aceptación: 9 de julio del 2012

${ }^{2}$ Enfermero. Correo electrónico: jaespinales12@gmail.com

${ }^{3}$ Enfermera. Labora en COOPEDANA R.L. Correo electrónico: gvivi00@,hotmail.com

${ }^{4}$ Enfermero. Asistente de la Comisión de Investigación de la Escuela de Enfermería de la Universidad de electrónico: dtinez@gmail.com

${ }^{5}$ Enfermero. Labora en el Hospital Rafael A. Calderón Guardia. Correo electrónico: charliebrown.cj@gmail.com

${ }^{6}$ Enfermera. Labora en la Clínica Oftalmológica. Correo electrónico: marilynqm@gmail.com

${ }^{7}$ Enfermera. Labora en PAIS-UCR. Correo electrónico:katvar95@hotmail.com
} 


\title{
Attitude assumed by nurses in regards to end of life decisions of people: Case of Costa Rica, 2011 ${ }^{1}$
}

\author{
Jerik Andrade Espinales ${ }^{2}$ \\ Viviana García Rojas ${ }^{3}$ \\ Daniel Martínez Esquivel ${ }^{4}$ \\ Carlos Miranda Otárola ${ }^{5}$ \\ Marilyn Quesada Morera ${ }^{6}$ \\ Katherine Vargas Villalobos ${ }^{7}$
}

Institution: University of Costa Rica

\section{CITED}

Andrade, J.; Garcia, V.; Martínez, D.; Miranda, C.; Quesada, M.; Vargas, K. (2012) Attitude assumed by nurses in regards to end of life decisions of people: Case of Costa Rica, 2011. Revista Enfermería Actual en Costa Rica. 23, 1-14. Avaible: http://www.revenf.ucr.ac.cr/voluntad.pdf.

\begin{abstract}
The research problem was to analyze what is the attitude assumed by nurses in regards to end of life decisions of people in Costa Rica during 2011. A quantitative exploratory and transactional methodology was developed, with a random sample and a subsample multistage taking into account the national hospitals Class A; obtaining a sample of 86 nursing professionals who completed a questionnaire. The obtained data was tabulated using a statistical package. The data demonstrated that although most of the participants were unfamiliar with the concept of end of life decisions, they related the said concept to the respect for the dignity, the rights and the autonomy of people in the same way they apply such ethical values when providing care. The research team concluded that the sampled nursing professionals favored the mentioned ethical values over their own personal ethics and morals.
\end{abstract}

Key Words: end of life decisions, Bioethics, moral, attitude, Nursing, care

\footnotetext{
${ }^{1}$ Date of reception: March 10, 2012

Date of acceptance: July 9, 2012

${ }^{2}$ Nurse. Private practice. E-mail: jaespinales12@gmail.com

${ }^{3}$ Nurse. COOPEDANA R.L. E-mail: gvivi00@hotmail.com

${ }^{4}$ Nurse. Assistant Research Committee of the School of Nursing at the University of Costa Rica: E-mail: dtinez@gmail.com

${ }^{5}$ Nurse. Rafael A. Calderón Guardia Hospital. E-mail: charliebrown.cj@gmail.com

${ }^{6}$ Nurse. Clínica Oftalmológica. E-mail: marilynqm@gmail.com

${ }^{7}$ Nurse. PAIS-UCR. E-mail:katvar95@hotmail.com
} 


\section{Revista Electrónica Enfermería Actual en costa Rica}

\section{INTRODUCCIÓN}

De acuerdo a Barrio, Lorda \& Júdaz (2004)

la voluntad anticipada de las personas sobre su cuidado al final de la vida, también conocida como testamento vital o instrucciones previas, nace en los Estados Unidos como un documento legal en el cual el individuo interesado dicta su testamento, de manera anticipada, sobre los cuidados que querría recibir al

final de su vida en caso de una enfermedad terminal (p.2).

Dilemas éticos como este y aspectos como el cuidado al final de la vida, las decisiones personales y familiares sobre los procesos de muerte y la participación de Enfermería se han abordado científicamente en países como España, Cuba, Estados Unidos, Holanda, Finlandia, entre otros; esto desde datos tanto cuantitativos como cualitativos. Se ha identificado que las personas que enfrentan procesos de muerte mantienen deseos claros respecto a su cuidado y que, aunque el perfil del profesional de Enfermería la posiciona como una disciplina de cuidado integral, en ocasiones la moral del personal entra en conflicto con las necesidades de los pacientes y sus familiares.

Es interesante observar el respeto que se demuestra hacia las decisiones al final de la vida en todos los países donde se han llevado a cabo discusiones al respecto. Existen varios estudios internacionales que han caracterizado el tema del cuidado al final de la vida y la respuesta del profesional de Enfermería. En Finnish nurses' interpretations of patient autonomy in the context of end of life decision making realizado en Finlandia por Hildén \& Honkasalo (2006), se analizó cómo el personal de Enfermería interpreta la autonomía de la persona ante una decisión anticipada al final de la vida. Las investigadoras concluyeron que existen tres discursos manejados por los profesionales de la disciplina: el discurso de apoyo, el analista y el práctico. El primero considera a la persona como un ser vulnerable y potencialmente inconsciente de las consecuencias de sus decisiones; el segundo plantea, que la persona puede equivocarse en sus decisiones por las influencias subjetiva, y el último, considera a la persona de manera neutral.

Otra de las investigaciones llevadas a cabo fue Nurses' attitudes towards end-of-life decisions in medical practice: a nationwide study in Flanders, Belgium por Bilsen, Mortier \& Deliens (2009). Su objetivo fue determinar la actitud de los profesionales de Enfermería ante las decisiones anticipadas al final de la vida y el rol de estos en dichas determinaciones. Se concluyó que la mayoría del personal de Enfermería acepta diferentes tipos de decisiones al final de la vida; la mayoría también opina que Enfermería debe participar activamente en el proceso de la toma de decisiones de los pacientes. Además, se determinó que la religión es uno de los aspectos más influyentes en la posición del personal de Enfermería en relación con las decisiones al final de la vida.

Esta aceptación se encuentra íntimamente relacionada con la cultura. En el caso de Costa Rica, es necesario preparar el campo de reflexión partiendo del contexto del profesional de Enfermería y su actitud sobre el tema. Además, se debe considerar la inexistencia de investigaciones relacionadas con el tema en el país. La relevancia del estudio de las actitudes reside en el hecho de que permite la predicción de conductas basándose en posiciones de las personas hacia cierto objeto social. "Cuanto más conocimiento se posea acerca de las actitudes 
de una persona en relación con determinados objetos, se tendrá una mayor certeza al hacer inferencias sobre su conducta" (Rodríguez, p. 44).

Asimismo, las instrucciones previas no se justifican como un derecho, ni por el dolor o el sufrimiento de la persona, sino que se involucra con el valor de la vida, la libertad, la dignidad e identidad personal; aspectos estrechamente relacionados con la Bioética. Esta última se define como:

la ciencia que regula la conducta humana, el comportamiento humano en el campo de la vida y la salud, a la luz de los valores y principios morales fundados en la dignidad de la persona humana. La Bioética constituye, por eso, una fuente de principios y normas de comportamiento que iluminan la conciencia y orientan a hacer elecciones siempre respetuosas de la vida y de su dignidad. De ahí, que sea una ciencia normativa, no sólo orientativa (Pardo, 2004, p.

16).

Cortina y Conill (2000) añaden que el fin de la Bioética es "señalar y fundamentar métodos de análisis ético que proporcionen pistas para que los propios implicados puedan vislumbrar la solución más correcta, justa, prudente o buena". (p.7)

Además, en Costa Rica, el aporte que puede hacer el profesional de Enfermería a la reflexión sobre la voluntad anticipada es válido y de gran importancia, ya que contribuye, desde su propia dimensión y hacia esta, a dicho debate ético y moral que involucra a estudiosos de múltiples disciplinas. El personal en Enfermería puede convertirse en una herramienta indispensable para brindar a las personas la máxima autonomía en sus últimos momentos de vida. El objetivo es que el paciente sienta apoyo y no autoridad por parte del personal sanitario, ya que este es el que más tiempo permanece al lado de la persona y su familia y, en consecuencia, el que conoce qué ayudas y obstáculos existen.

El tema en cuestión ha hecho reflexionar a profesionales de la salud, desde diferentes perspectivas, acerca de la manera cómo se lleva a cabo este proceso, pues los requisitos son mínimos; la persona debe ser mayor de edad, capaz de expresarse y ser libre para poder ejercerlo. En la práctica, sin embargo, no es tan simple como parece. Lo anterior ha generado importantes investigaciones alrededor del mundo sobre el cuidado de las personas en proceso de muerte. Además, ha motivado el estudio de la temática en Costa Rica a partir de la actitud del profesional de Enfermería respecto al tema.

Según lo expuesto, resulta novedoso analizar el trasfondo del pensamiento de Enfermería, como ente de cuidado de las personas al final de la vida, en un país como Costa Rica, donde el concepto de voluntad anticipada en los operadores sanitarios es poco conocido. A partir de lo anterior, se planteó el siguiente problema de investigación: ¿Cuál es la actitud que asume el y la profesional de Enfermería frente a la voluntad anticipada sobre el cuidado de las personas al final de la vida: caso de Costa Rica, 2011? A partir de esta interrogante, se plantea el objetivo de analizar la actitud que asume el y la profesional de Enfermería frente a la voluntad anticipada sobre el cuidado de las personas al final de la vida: caso de Costa Rica, 2011. 


\section{Materiales y métodos}

La población estuvo conformada por el personal de Enfermería que labora en los hospitales nacionales clase A de la Caja Costarricense de Seguro Social.

La investigación se enmarcó dentro de un enfoque cuantitativo con un diseño no experimental. Es un estudio transeccional exploratorio, según Hernández-Sampieri, Fernández-Collado y Baptista-Lucio (2007) “se trata de una exploración inicial en un momento especifico". (p.142)

Se llevó a cabo un muestreo probabilístico por conglomerados y un submuestreo polietápico posterior. El muestreo sistemático quedo conformado por el Hospital Rafael Ángel Calderón Guardia (HCG) y el Hospital San Juan de Dios (HSJD); la muestra final, por 40 profesionales del HCG y 46 del HSJD.

Estos profesionales debían estar inscritos y al día en las obligaciones del Colegio de Enfermeras y Enfermeros de Costa Rica, ser graduados con un grado o posgrado en Enfermería y laborar en los servicios que fueron seleccionados al azar (Maternidad, Supervisión de Enfermería, Cirugías y Medicinas). Cada participante del estudio debió completar el consentimiento informado diseñado por la Vicerrectoría de Investigación de la Universidad de Costa Rica.

La recolección de los datos se realizó por medio de un cuestionario de 21 preguntas: 10 cerradas, 5 semiabiertas y 6 abiertas, de acuerdo con el sistema de variables planteado.

El análisis de los datos se llevó a cabo mediante el paquete estadístico SPSS 17.0; se examinaron con estadística descriptiva y se realizó la distribución de las frecuencias con su respectivo porcentaje de los datos cuantitativos. Todos los datos se confrontaron para determinar divergencias, congruencias y coincidencias entre ellos. Todo esto permitió analizar la información, recopilada por medio del cuestionario, para verificar su confiabilidad e interpretar y comprender los resultados.

\section{Resultados}

Se consideró importante para la investigación caracterizar a los participantes; se obtuvo los siguientes resultados. En el rubro edad, la mayoría de participantes se encuentra en un rango de entre los 23 y los 27 años, lo cual representa un 23,5\% del total de la muestra. La mayoría son de sexo femenino; un 77,6\%. En cuanto al rubro de nacionalidad, un $98,8 \%$ de los participantes son costarricenses. Por último, un $71,8 \%$ posee el grado académico de Licenciatura.

Con respecto al servicio hospitalario en el que laboran, la mayoría de participantes trabaja en el Servicio de Cirugía, 40 de las respuestas, mientras que en segundo lugar, con 24 respuestas, se encuentra el Servicio de Medicina.

Finalmente, con respecto a los años de experiencia profesional, la mayoría de participantes tiene de 6 a 10 años de experiencia laboral, un $29,4 \%$ de las respuestas.

En cuanto a la identificación del concepto de voluntad anticipada, se indagó acerca del conocimiento que se manejaba acerca de este. Un 54,7\% de los entrevistados dijo no conocer el término, mientras que un 45,3\% afirmó conocerlo. Al solicitarles una definición, las respuestas fueron diversas, aunque la mayoría de los participantes relacionó el concepto de voluntad anticipada con principios tales como la autonomía, el derecho y la voluntad de las personas sobre su cuidado. 
Al consultarles sobre su posición con respecto a la voluntad anticipada, 60 de las respuestas mostraron una posición favorable, mientras que 26 fueron neutras. Además, se interrogó acerca de los conceptos que consideraban relacionados con la voluntad anticipada y se obtuvieron distintos resultados. Resalta el hecho de que la mayoría los participantes la relacionan con una manera de preservar la dignidad de la persona (58 respuestas) y con un medio de promover la autonomía de la persona (47 respuestas).

Al analizar la integración de los principios de Bioética, se consultó a los participantes sobre la definición del concepto de. Se destaca que un 37\% la definió como una ciencia que busca respetar la vida del ser humano; el resto la relacionó con la moral y con normas y reglamentos que definen la conducta.

Al consultarles sobre los principios que consideran parte de la Bioética, en primer lugar, 59 de las respuestas consideran que la dignidad es el principio que se relaciona más con la Bioética, seguido de la justicia, con 51 respuestas, y de la autonomía, con 50 .

Para analizar el fundamento moral del profesional en Enfermería en Costa Rica, se indagó sobre aspectos que influyen en la moral de las personas, esto tomando como base el concepto de moral y sus determinantes personales y sociales.

Al consultarles sobre los determinantes que influían sobre su práctica profesional, se expusieron aspectos religiosos, políticos, personales y de cualquier otra índole que formara parte de las costumbres, hábitos y valores culturales de los individuos, y que, a criterio personal, interviniera en el pensamiento y el hacer profesional.

La mayoría de participantes $(61,2 \%$ y $54,1 \%)$ considera que los elementos más influyentes son los personales y los religiosos, respectivamente.

Por último, para identificar el rol del profesional de Enfermería en Costa Rica en la experiencia de la persona respecto a la voluntad anticipada sobre su cuidado al final de la vida, se indagó sobre las actividades que los profesionales relacionan con el cuidado.

Según las repuestas obtenidas, las principales fueron el cuidado directo, la educación y las relaciones interpersonales, seguidas por la administración de medicamentos, las actividades de higiene y comodidad e incluso otras como la nutrición y la rehabilitación identificadas en menor medida.

También, se indagó sobre los roles que habían desempañado los participantes en el cuidado de personas en proceso de muerte. Se obtuvo, en primer lugar, el rol de apoyo con 81 respuestas, seguido del rol de ayuda y el de educador con 67 y 62 respuestas, respectivamente.

Por último, se indagó cuáles roles consideran que se deberían cumplir. A esto respondieron principalmente: rol de cuidador, educador y servidor, también señalaron los papeles de mediadores e investigadores. Algunos de los participantes consideraron que no les corresponde ningún rol y otros identificaron roles como el apoyo y soporte al paciente para enfrentar su enfermedad en estado terminal e intervenir en la crisis al final de la vida.

\section{Discusión}

La participación de todas y todos los profesionales en Enfermería se consideró importante para el desarrollo de este estudio. Sin embargo, al analizar las características sociodemográficas más relevantes resaltan 


\section{Revista Electrónica Enfermería Actual en costa Rica}

los siguientes aspectos: La mayoría de participantes eran adultos jóvenes, esto significa que la profesión de Enfermería en nuestro país vive un cambio generacional importante. Los participantes, como menciona Malvárez (2007), "se han desarrollado en la era de la información y la tecnología, la cual ha impactado la fluidez de la comunicación científica de calidad" (p. 522). El sexo femenino continúa dominando la disciplina, debido a hechos históricos que marcaron su desarrollo. Además, los participantes poseían, como mínimo, el grado académico de Licenciatura, esto debido a los criterios de inclusión de esta investigación y a lo estipulado en la Ley General de Salud en el Artículo 40.

La mayoría de participantes labora frecuentemente en los servicios de Cirugía y Medicina. Según el Área de Estadística en Salud de la Caja Costarricense de Seguro Social, estos servicios son los que más personas atienden y, por ende, los que más recursos humanos demandan. (Caja Costarricense de Seguro Social, 2009)

El cambio generacional también se ve reflejado en los años de experiencia del personal. Por un lado, este ha sido partícipe del desarrollo del área de investigación de la Enfermería, lo cual pudo tener un impacto favorable en el desarrollo de este estudio. Por el otro, los demás participantes han acumulado experiencias que moldearon el concepto de Enfermería reflejado en las respuestas de este proyecto.

El desarrollo del tema de la voluntad anticipada es reciente en Latinoamérica, primero en México y luego en Argentina. En Costa Rica, es todavía novedoso; esto se demuestra en las respuestas sobre el conocimiento acerca del término.

En la definición de voluntad anticipada dada por Barrio, Lorda y Júdez (2004), se identifican valores implícitos tales como la voluntad, la autonomía y los derechos de las personas. Algunos de los participantes lograron relacionar estos valores, en un intento por definir dicho concepto. No cabe duda que la reflexión de estos parte de su individualidad; cada una de las respuestas se justifica por los niveles de reflexión ética.

Partiendo de la reflexión moral según Hermensen \& Henk (2005), el pensamiento se basa en la formación personal y profesional; este nivel se centra en juicios de valor sobre cómo operar en la toma de decisiones relacionadas con la vida y el final de ella. Desde la ética normativa, se intenta unificar conceptos, principios, normas y razonamientos (Beauchamp, 2001). En la ética descriptiva, se parte de las tradiciones morales y se utilizan bases científicas para explicar el razonamiento. Por último, desde la metaética, el razonamiento establece la existencia de juicios, valores y definiciones a los cuales se les confiere valor. (Vargas, et al., 2010)

A partir de la reflexión realizada sobre el concepto de voluntad anticipada, se expone una particularidad en el estudio: a pesar de que la mayoría menciona no conocerlo, esta y aún más participantes se muestran a favor de dicho proceso. 
Solange (s.f.) explica que dicha singularidad puede deberse a un proceso de toma de decisiones, el cual se justifica en los métodos de toma de decisiones. En el método principialista, se pretende respetar cuatro principios, la autonomía y la beneficencia, los principios máximos que se deben respetar, y la justicia y la no maleficencia, los mínimos que se debe respetar (Beauchamp y Childress, 1987). Por otro lado, en contra de la universalidad del método principialista, se encuentran el método casuístico, el cual analiza cada caso como un hecho particular, y el método narrativo, que aborda cada uno como si se tratara de un proceso. Estos métodos toman en cuenta todos los determinantes que influyen en la toma de decisiones ante un dilema bioético como la voluntad anticipada (Solange, s.f.)

Los participantes consideran que los dos conceptos más relacionados con la voluntad anticipada son la dignidad y la autonomía. Menciona Valverde (2005) que "la dignidad procura ejercer la libertad del ser humano, esto al suponer que las personas no deben ser tratadas como objetos" (p. 171). Por su parte, "la autonomía considera los valores y creencias de la persona como la principal consideración moral en la determinación de las responsabilidades morales del profesional en la asistencia a las personas" (Beauchamp, 1987, s.p.). Ambos conceptos se encuentran íntimamente relacionados con el respeto por la voluntad de las personas y le otorgan un valor especial a la vida de quienes sufren de un proceso en el final de esta.

Para analizar el tema de la voluntad anticipada, fue importante integrar la Bioética como "ciencia que ilumina la toma de decisiones respetuosas a la vida y a la dignidad" (Pardo, 2004, p. 16). La mayoría de los participantes define la Bioética como una ciencia que busca respetar la vida; los profesionales consultados poseen claridad sobre el objeto de la Bioética como ciencia que busca respetar los derechos y la dignidad de las personas. Por otro lado, un grupo importante relaciona dicho concepto con la moral o con un conjunto de normas que guían la conducta de las personas. Tealdi (2008) y Pardo (2004) mencionan que estos aspectos están relacionados con la Bioética siempre y cuando se enfoquen en el respeto a la vida.

Además, los participantes reconocen la dignidad como el principio más importante de la Bioética, lo cual deja en segundo plano los "principios de Bioética" más populares, como lo son la justicia, la autonomía, la beneficencia y la no maleficencia. Se observa cómo los profesionales en Enfermería que participan del estudio dotan a los seres humanos de dignidad, independientemente del proceso de salud-enfermedad que esté viviendo. Menciona Valverde (2005) que

"la dignidad le da a la persona identidad, lo cual la vuelve única en el universo y le otorga un valor especial a la vida. La dignidad, la identidad y el valor de la vida son los postulados que la Bioética busca defender" (p. 85). 
Los principios bioéticos responden a métodos de toma de decisión, en Bioética, que ayudan a lograr una mayor corrección en los juicios realizados y las recomendaciones que se llevan a cabo, esto por las situaciones de conflicto entre valores, principios, derechos u otros tipos de normas que se presentan. Según Solange, el método que predomine en las actitudes y acciones del profesional dependerá de su formación, criterio y sistema de valores. Según las respuestas, se refleja el rechazo al universalismo del método principialista y se abre la puerta a otros métodos de toma de decisión como el casuístico y el narrativo.

Por otro lado, el modo en el que las personas enfrentan la enfermedad y administran la salud está influido por su marco de valores ético-religiosos; algunos profesionales señalaron que la religión es fuente de principios y valores, los cuales permiten fundamentar su práctica y realizar juicios de valor sobre las conductas.

La religión es un aspecto importante por varias razones. Primero, porque, históricamente, nuestro país se ha desarrollado en un plano religioso; segundo, por el significado que las religiones le dan a la vida y a la muerte. En última instancia, es relevante, pues, las religiones son, por sí solas, un conjunto de normas éticas y morales.

La influencia de la religión, específicamente la del Cristianismo, ha implantado principios incuestionables acerca del funcionamiento de la sociedad e implementado concepciones sobre fines jerarquizados y obligatorios en la aplicación de la ética. La religión predominante en Costa Rica, la Católica Romana, se caracteriza por ser revelada; esto significa que existe una serie de recomendaciones o mandamientos que rigen la toma de decisiones de las personas.

Gran parte de los participantes reconoció que, en su práctica profesional, son muchos los factores personales que influyen en sus decisiones al momento de realizar algún procedimiento sobre las personas bajo su cuidado. La mayoría de los profesionales de Enfermería se refirieron a aspectos tales como las experiencias vividas, la empatía y la posición con respecto a la vida.

La experiencia como modulador de la moral del profesional se justifica al hacer referencia a un hecho ya pasado, como lo menciona Pieper (1995), el utilizar sucesos ocurridos como punto de partida para demostrar la validez de un juicio o acción es la forma más corriente y típica de fundamentación moral. Este autor también explica que, con frecuencia, se recurre a un sentimiento para fundamentar la justicia o juicio acerca de un acto, es decir, se aducen estos sentimientos y sensaciones como motivo de una determinada conducta. Por lo tanto, la acción que se trate queda, hasta cierto punto, explicada y resulta comprensible.

La política, como una tradición, puede ser parte de la moral y ser significativa su forma de expresarla. Se distingue de la ética, y por consiguiente de la moral, ya que puede ser fundamentada con validez siempre y cuando sea por medio de la comunicación cotidiana e intercambio de argumentos. En el caso de los participantes en cuestión esta no fue relevante.

Entre otros aspectos que los individuos piensan que intervienen en su práctica laboral, destacan el conocimiento teórico, el género y el aspecto económico. Se observó las diversas influencias que giran en torno al quehacer profesional de Enfermería, ya que para toda acción existe una serie de motivos de distinto origen que, 
generalmente, son combinados para justificar decisiones, juicios o acciones. Estos, sin embargo, adquieren reconocimiento hasta que exista un consenso entre todas las partes involucradas en la toma decisiones.

Según las actividades del cuidado de Enfermería identificadas por los participantes, se puede afirmar que la intervención del profesional en Enfermería en Costa Rica, con respecto a las experiencias de salud, se relaciona principalmente con la satisfacción de las necesidades biológicas, $\mathrm{y}$, en menor grado, las sociales y espirituales. Esto puede estar relacionado con lo que menciona Siles (2000) sobre la expresión del cuidado relacionado a la cultura, tanto de la persona que brinda el cuidado como de quien lo recibe, y especialmente a la cultura con la que se forman los enfermeros y enfermeras, una cultura curativa.

Al ser el método principialista el primero en la toma de decisiones en Bioética, ha influido mucho y continúa teniendo injerencia sobre el ejercicio profesional de distintas disciplinas. Ejemplo de esto es el hecho de que, dentro de las respuestas de Enfermería, se incluyan actividades enfocadas en la curación, tales como el cuidado directo, la administración de medicamentos, la higiene y la rehabilitación.

A pesar de que, como menciona Tealdi (2008), "el cuidado y restitución de la salud requieren el binomio del acto médico-asistencial y del cuidado espiritual" (p.92), por diversas razones como prejuicios, creencias, valores, competencias, temores sobre el qué dirán, tiempo, número de personas disponibles o costos, entre otras, actualmente se tiende a limitar el cuidado al aspecto corporal del ser humano, lo cual deja de lado la atención a lo espiritual, elemento que permite lograr la unicidad del ser; la integración de cuerpo-alma en una unidad indisociable.

Según los roles que los participantes han cumplido en el cuidado de personas en proceso de muerte, se observa que resulta cada vez más común que el personal de Enfermería reconozca que el enfermo terminal es el principal protagonista de sus cuidados una vez que se le brinden las herramientas pertinentes, tales como la educación y la información.

Pavlish y Ceronsky (2009) coinciden en que algunos de los roles del profesional de Enfermería en el cuidado al final de la vida son mejorar el crecimiento personal de la persona, responder a los sentimientos generados y apoyar a la familia. Lo anterior se debe a que es, específicamente durante este proceso, cuando se genera más dudas y estrés; los sentimientos de culpa, impotencia depresión y ansiedad aumentan, lo cual complejiza la situación estas personas.

Al indagar en los roles que idealmente deberían de cumplir los profesionales en Enfermería, resaltó el del cuidado, elemento inherente al quehacer de la Enfermería. Tal como lo menciona Sanabria (2002): "El centro de interés de la Enfermería está en los cuidados de la persona que, en interacción continua con su entorno, vive experiencias de salud" (p. 4).

El significado que le brindan este y los demás roles al cuidado al final de la vida pretenden conocer quién es la persona, cuáles son sus sentimientos, qué desea y qué necesita. Lo anterior, al compartirlo con otras disciplinas, puede mejorar sustancialmente la calidad de vida del individuo, pues en muchas ocasiones, a las personas que se encuentran al final de su vida se les niega el derecho a opinar, pues se olvida que, como el resto, tienen deseos, sentimientos y necesidades de expresarse y ser escuchados.

Tealdi (2008) se refiere al Decálogo de los Derechos del Enfermo Terminal, el cual surge con el fin de lograr satisfacer las necesidades que tienen las personas en un proceso del final de la vida. Entre ellas, menciona 
las necesidades biológicas, como el control del dolor; necesidades emocionales para el manejo de sus angustias, temores; necesidades espirituales en el soporte de sus creencias religiosas y en sus inquietudes existenciales, y necesidades socio familiares de soporte por sus seres queridos, una comunicación cálida, afectiva y permanente, y de tomar decisiones compartidas teniendo acompañamiento hasta el final (p. 494). En todas estas necesidades, los profesionales en Enfermería son capaces de desarrollar roles que marquen una diferencia en el cuidado al final de la vida.

Se concluye que, a pesar de que algunos participantes de esta investigación refirieron no conocer el concepto de voluntad anticipada, otros declararon mantener una posición favorable hacia este. A pesar de las definiciones, demostraron tener noción acerca del tema.

La mayoría de los involucrados propusieron definiciones de Bioética a través de la mención de aspectos como la ética, la ciencia, la dignidad y la moral; estos pueden ser considerados en la voluntad anticipada de las personas de nuestro sistema de salud. En cuanto a los procesos de Enfermería en los cuales se reflejan los principios bioéticos, la mayoría de los participantes hizo referencia a procesos relacionados con el cuidado al final de la vida.

Además, la gran mayoría tuvo experiencias en el cuidado al final de la vida y señaló, como influencias principales en su práctica, los aspectos religiosos y personales, tales como las emociones, la ideología con respecto a la vida y las situaciones vividas. Según los participantes, entre los roles que desempeña la Enfermería en el cuidado de personas al final de la vida destacan los siguientes: el de apoyo, el de ayuda y el de educación. En cuanto a los papeles que debería asumir, destacan el de cuidador, educador y servidor.

\section{Conclusiones}

- La mayoría de los y las profesionales en Enfermería encuestadas refirió una posición favorable hacia la voluntad anticipada y, a pesar de las divergentes definiciones, demostraron tener noción sobre el tema.

- La mayoría de los y las profesionales proponen definiciones de Bioética a través de la mención de aspectos como la ética, la ciencia, la dignidad y la moral. Los elementos identificados con mayor frecuencia como principios de la Bioética por parte de los encuestados fueron la dignidad, la justicia y la autonomía.

- A pesar de la influencia del método principialista, las y los encuestados no identificaron estos principios con tanta frecuencia como lo hicieron con la dignidad. El ejercicio de la Enfermería se aparta del paradigma biomédico reflejado en el método principialista tomando en cuenta principios como la dignidad, la equidad y la autodeterminación.

- Estos principios y postulados, que pueden ser considerados en la voluntad anticipada de las personas de nuestro sistema de salud, son un acercamiento a un cambio de pensamiento de una Bioética latinoamericana que pueda basarse y fundamentarse en las necesidades y realidades sociales y culturales propias de nuestra región.

- Las principales influencias que, según los profesionales, influyen en su práctica, tienen que ver con aspectos religiosos y aspectos personales, como las emociones, la ideología con respecto a la vida y las experiencias. 
- Entre los roles que desempeña la Enfermería, según la experiencia de los encuestados en el cuidado de personas el final de vida, están el rol de apoyo, el de ayuda y el de educador. Por otra parte, entre los roles que según los mismos encuestados debería asumir Enfermería en el cuidado al final de la vida, se destacaron el de cuidador, educador y servidor.

- Esta investigación significó un primer acercamiento a un terreno todavía árido en nuestro país. Se considera que se debería continuar realizando nuevas investigaciones bajo el mismo enfoque cuantitativo, pero con variaciones en el tipo de estudio, esto con el objetivo de profundizar aún más en la temática.

\section{Agradecimiento}

A Dios, a nuestras familias, a nuestros amigos y amigas, a los profesores y profesoras, y al comité asesor de este trabajo, Doña Ligia, Don Rafael y Don Manuel.

Gracias a todos y todas por guiarnos por el camino correcto, por el tiempo invertido y por enseñarnos con tanta sabiduría, pero principalmente gracias por creer en nosotros. Siempre les recordaremos en nuestro corazón.

Grupo de Seminario

\section{Referencias}

Asamblea Legislativa de la República de Costa Rica (1973) Ley General de Salud. Título I: Derechos y deberes concernientes a la salud personal. Artículo 9. San José, Costa Rica.

Barrio, I., Lorda, P., y Júdez, J. (2004). De las Voluntades Anticipadas o Instrucciones Previas a la Planificación Anticipada de las Decisiones. NURE Investigación. Número 2. (s. p.).

Beauchamp, T. (1987). Ética Médica. Las responsabilidades morales de los médicos. Barcelona, España: Editorial Labor.

Beauchamp, T. L., y Childress, J. F. (2001). Moralidad y justificación moral. En T. L. Beauchamp, \& J. F. Childress, Principios de Ética Biomédica. Barcelona: Masson.

Bilsen, J., Mortier, F., \& Deliens, L. Nurses' attitudes towards end-of-life decisions in medical practice: a nationwide study in Flanders, Belgium. Palliative Medicine, 23 (7), 649-658.

Boff L. (2004). Ética y moral. La búsqueda de los fundamentos. España: Editorial Sal Terrae. 
Cabrera, J. (2005). Temas de Bioética. Del inicio al final de la vida humana. San José, Costa Rica: Editorial Universidad Estatal a Distancia.

Cortina, Adela. (1999). Ética. Madrid, España: AKAL.

Cortina, A. (2000). Ética mínima. Recuperado de: http://www.yovanyospina.com/asignaturas/ETICA/ETICA\%20DE\%20LOS\%20MINIMOS\%20ADELA\%20CO RTINA.pdf

Cortina, A y Conill, J. (2000). Diez palabras clave en ética de las profesiones. Pamplona, España: Verbo Divino.

Drane, J. (1999). El cuidado del enfermo terminal. Ética clínica y recomendaciones prácticas para instituciones de salud y servicios de cuidados domiciliarios. Washington, D.C: Organización Panamericana de la Salud.

García C., Cruz, F., Muñoz, A., Montoya, R. (2010) Influencia de las emociones en el juicio clínico de los profesionales de la salud a propósito del diagnóstico de enfermedad terminal. International Journal of Clinical Health \& Psychology, págs. 57-73.

García, A., Abad, E., González, Ma., y Sánchez, R. (2006) La planificación anticipada de las decisiones al final de la vida: el rol de los profesionales sanitarios en general y de Enfermería en particular. NURE Investigación. 20: s.p. Recuperado de:

http://www.nureinvestigacion.es/ORIGINALes_detalle.cfm?id_ORIGINAL=96\&id_menu=135

Hermensen, M., \& Henk, A. (Diciembre de 2005). Palliative care teams: Effective through moral reflection. Journal of Interprofessional Care, vol. 3 y num 5, 561-568.

Hernández, R; Fernández, C. y Baptista, P. (2007). Fundamentos de metodología de la investigación. Madrid, España: McGraw-Hill Interamericana.

Hildén, H., Louhiala, P., Honkasalo, M., \& Palo, J. (2004). Finnish nurses' views on end of life discussions and a comparison with physicians' views. Nursing Ethics. (11) (2), 165-178. 
Hildén, H., \& Honkasalo, M. (2006). Finnish nurses' interpretations of patient autonomy in the context of end of life decision making. Nursing Ethics 13(1), 41-51.

Pardo, J. (2004). Bioética práctica al alcance de todos. Madrid, España: Ediciones RIALP.

Paulish, C y Ceronsky, L. (2007). Nurses perceptions About Palliative Care. Oncology Nursing Forum, (34) (4), 793-800.

Pavlish, C., \& Ceronsky, L. (agosto, 2009). Oncology Nurses' Perceptions of Nursing Roles and Professional Attributes in Palliative Care. Clinical Journal of Oncology Nursing, vol y número, 404-412.

Rodríguez, A. (1976). Psicología social. Traducido por Crespo A. México: Editorial Trillas.

Sgreccia, E. (1996). Manual de Bioética. D.F., México: Editorial Diana.

Siles, J. (2008) Historia de la Enfermería, una aportación epistemológica. . Revista Cultura de los Cuidados número 24, 5-6 Recuperado de: http://www.index-f.com/cultura/24pdf/24005006.pdf

Scheler, M. (s.f.) Ética formal y ética natural de los valores. Revista de Occidente. Volúmen 2, pp. 310.

Solange, C. (s.f.) Introducción a la Bioética aplicada. En: Programa de Educación Permanente en Bioética. Introducción a la Bioética clínica y social. (Págs. 8-11, 21-22 31, 35-36).

Tealdi, J. (2008). Diccionario Latinoamericano de Bioética. Colombia: Universidad Nacional de Colombia: UNESCO.

Valverde, F. (2005). La Bioética. Ética y Salud, un enfoque ético para trabajadores de la salud. Ed. de la Universidad Estatal a Distancia (UNED). San José, Costa Rica. 60-88, 171. 